How to reference this article

Pieri, M. (2019). Il pubblico femminile a teatro in età moderna. Italica Wratislaviensia, 10(2), 37-49.

DOI: http://dx.doi.org/10.15804/IW.2019.10.1.15

\author{
Marzia Pieri \\ Università degli Studi di Siena \\ marzia.pieri@unisi.it \\ ORCID: 0000000231695277
}

\title{
IL PUBBLICO FEMMINILE A TEATRO IN ETÀ MODERNA
}

\section{WOMEN AT THE THEATRE IN THE MODERN ERA}

\begin{abstract}
At the dawn of the modern era, aristocratic women, whether at court or in the academy, shed a noble aura on the new classic theatre and were often the privileged recipients of dedicated plays and poetry essays. They were totally different from the theatre-going women that bought tickets in $16^{\text {th }}$ - and $17^{\text {th }}$-century Europe. Women in the theatre, whether on the stage or in the audience, were a problem and a source of scandal for Catholic and Protestant authorities, who wanted to ban them from attendance. However, their presence kept growing and finally established itself in the $18^{\text {th }}$ century, when women became the main heralds of good taste and sentiment. This essay discusses the two aspects of this story.
\end{abstract}

Keywords: spectator, censorship, patronage, comedy, sensitivity 
Sulla scorta della semiotica e della teoria letteraria della ricezione Sè ormai riconosciuta l'idea che esista anche una drammaturgia dello spettatore, che concorre a scrivere lo spettacolo alla pari dell'autore, dell'attore e poi del regista. La storia materiale e culturale del pubblico teatrale costituisce per gli studi una fonte metodologicamente importante e ancora trascurata e un bacino documentale assai promettente. $\mathrm{Ha}$ a che fare con una semiosfera complessa di relazioni corporee, con le diverse modalità culturali della visione; investe il senso stesso dell'esperienza teatrale e i legami che in ogni epoca la collegano con la storia circostante. Se dello spettatore di ancien régime sappiamo poco, la spettatrice è ancora più sfuggente, e resta una comparsa secondaria, ostaggio tradizionale di due opposte fazioni: ci sono, in maggioranza, i proibizionisti che tendono a escludere e a temere il suo diritto al piacere e all'evasione, giudicandola solitamente una preda (emotivamente vulnerabile e corruttibile) da difendere da se stessa e dagli altri, e i progressisti, che invece le concedono un accesso protetto in platea con degnazione paternalistica e molti distinguo. Sulle implicazioni teatrali della peculiare sensibilità femminile si gioca la partita fra i più che la stigmatizzano come potenziale inclinazione peccaminosa, e i meno che la considerano un possibile valore aggiunto. Fra le spettatrici in carne ed ossa cinque, sei e settecentesche e le spettatrici ideali a cui alludono in vario modo i testi drammaturgici e i trattati di poetica coevi c'è comunque una totale discontinuità.

Per occuparsi delle prime bisogna far riferimento a una serie di elementi materiali che riguardano le modalità concrete in cui si produceva e si consumava l'esperienza del teatro in epoche e luoghi diversi della storia: gli spazi e le occasioni, l'economia, l'organizzazione del tempo festivo. Le spettatrici ideali, invece, sono una presenza astratta nella teoresi teatrale, chiamate sempre in causa pretestuosamente negli snodi estetici più controversi. L'archetipo del teatro/lupanare ossessiona l'immaginario occidentale da molti fronti: la promiscuità e la libertà di una massa incontrollabile che si diverte costituiscono sempre un problema che sollecita controlli e censure. Non è solo il carnevale ad essere pericoloso, ma qualunque occasione in cui ci si riunisce in pubblico, a cominciare dagli appuntamenti religiosi. Già San Bernardino da Siena, ad 
esempio, nelle sue prediche (fruite da un pubblico di maschi e di femmine rigorosamente separati da transenne e gradinate, come ci documentano vari dipinti) si interrompeva in continuazione per richiamare all'ordine i molti che si distraevano nell'inevitabile gioco del corteggiamento a distanza. In area protestante nord-europea domina l'associazione fra teatro, lascivia e crapula: le maschere, la musica e la danza "apostare faciunt sapientes" e una ricca iconografia ripercorre ossessivamente scene festive e teatrali in cui le donne presenti sono soltanto puttane, le spettatrici alla pari delle attrici; il grande capitolo europeo del teatro gesuitico è fatto esclusivamente di maschi, e ancora nell'illuminata Arcadia sono in molti a difendere un teatro di soli attori uomini per un pubblico composto in prevalenza da uomini, affiancato da minori e privati teatri femminili di collegio e di convento previsti per le signore.

Vorrei tuttavia prescindere qui dalle molte declinazioni possibili degli sguardi sessuofobici appuntati sui corpi femminili esposti in palcoscenico, per esplorare invece rapidamente un'altra linea di pensiero,per cui, al contrario, la presenza delle donne quali destinatarie o madrine dello spettacolo vale simbolicamente da marchio di qualità che riscatta il "basso" del teatro in termini morali e culturali. Un fenomeno che di solito si produce in momenti di discontinuità o di rifondazione. La professione di filoginia, del resto, da Boccaccio in poi, vale sempre come segnale positivo di rottura della tradizione, e non implica affatto, va ricordato, una corrispondente emancipazione reale delle donne per cui si dichiara di voler scrivere.

Cominciamo dal principio, cioè dalla festa signorile rinascimentale, che incuba e genera la rappresentazione drammatica agli inizi del sedicesimo secolo. Siamo in una sfera elitaria e privilegiata ma anche molto sperimentale e in un momento storico in cui il teatro è ancora tutto da inventare e da riconoscere, in modalità materiali spesso avventurose e disagevoli. Si tratta di fondare i parametri-base di un'esperienza totalmente nuova per un pubblico-cavia precettato dal principe, che deve imparare ad essere tale, e che, abituato all'ascolto distratto di musiche e frammenti performativi inseriti nel banchetto e nella festa, subisce con disagio la scomoda e prolungata visione di spettacoli statici, poco comprensibili, troppo lunghi (da 2 a 6 ore), fruiti su gradinate, panche e tri- 
bune affollate. Durante i famosi festival plautini di Ercole I a Ferrara, ad esempio, gli ospiti del duca furono sottoposti a notevoli stress: sappiamo che durante la recita dei Menaechmi imperversarono i borsaioli, o che, dopo l'interminabile recita dell'Eunuco che costrinse gli astanti a una lunga immobilità, la sala puzzava in modo intollerabile:

Remaxe una tal puza de tanpho et de pisso del tanto pissare che haveno fatto quelle donne, per modo che tucto hogi lo Ill.mo S. D. ha bisognato perfumare la dicta salla. (Coppo, 1968, p. 52)

Molto più liberi, festosi e scollacciati erano i trattenimenti vecchio stile - mescolati di sketch comici, di danze e di musiche - che per tutto il Cinquecento sopravvivono all'avvento del teatro rappresentativo di marca classicistica. I grandi appuntamenti che segnano il faticoso decollo della cosiddetta commedia erudita a corte coesistono a lungo con le feste licenziose d'antan, farcite di scherzi grossolani e allietate dalla presenza di prostitute, nei palazzi romani di Alessandro VI e di Leone X, come in quelli veneziani di cui ci racconta, spesso scandalizzato, Marin Sanuto nei suoi diari, per esempio il 26 febbraio 1519:

In questo carlevar non si pol far mascare, ma fase caze per campi et comedie di zentilhomeni et altri in caxe, dove intravien putane solamente ben vestite, et stano a balar etc.; cosa, per opinion mia, vergognosa a questa ben instituta Republica. (Sanuto, 1879, XXXVI, p. 501)

Oppure il 14 febbraio 1525 osserva che le cattive abitudini sono passate dai padroni ai servitori:

In questa sera, a Santa Maria Formosa, in la caxa sul ponte da cha' Morexini, per una compagnia de famegii de zentilhomeni fu fatto una festa et balli. Quali messeno un ducato per homo, feno un signor, e tutti con la sua putana, et ballono tutta la notte et cenono lì, né volse alcun ve intrasse. Siché, a concorentia di nobili, li famegii fanno festa. Fo mal fatto, et li Cai di X dovea proveder. (Idem, 1879, XXXVII, p. 578)

La differenza e la qualità dei trattenimenti sono dunque marcate dalla presenza e dal patrocinio attivo delle principesse padrone di casa: 
Isabella d'Este, Eleonora Gonzaga, Lucrezia Borgia e le dame dei loro entourage impongono decoro e buona creanza, e testano la qualità della festa nuziale o diplomatica, a cui partecipano spesso come danzatrici o recitanti e in cui si incastona sempre più spesso la recita della commedia. La sociabilità aristocratica assorbe il teatro all'interno dei suoi riti mondani come prolungamento della conversazione ed acme dell'appuntamento festivo sotto il segno dell'allegoria amorosa, e la «dama di palazzo» celebrata da Castiglione nel quarto libro del Cortegiano ne è garante e custode. La recita moderna di commedie, e poi anche tragedie e pastorali, inventata nelle corti italiane cinquecentesche si svolge all'ombra di un tale pubblico femminile di élite entro una griglia di riferimento cortese e neo-platonica, che ancora lambisce la seicentesca civiltà della conversazione e i codici della galanteria nei salon parigini del diciottesimo secolo.

Il prototipo di questa sociabilità teatrale aristocratica si può riconoscere in una celebre e fortunatissima commedia senese, Gl'Ingannati, scritta e recitata dagli Accademici Intronati per le gentildonne della città, e allestita il martedì grasso del 1532 nel Palazzo Pubblico: lo spettacolo è offerto loro come risarcimento e palinodia di un precedente rito festivo misogino celebrato all'inizio del carnevale, in cui i giovani accademici, stanchi di essere respinti, avevano collettivamente abbandonato il culto di Venere e sacrificato a Minerva i pegni d'amore ricevuti dalle rispettive dame in uno spettacolare pageant allegorico e musicale. Ma poi si erano pentiti. La commedia a cui essi consegnano la propria ritrattazione è inseparabile da questa cornice, e infatti le numerose stampe che la conservano si intitolano Il sacrificio e non Gl'Ingannati; il prologo (come quasi tutti i prologhi delle commedie rinascimentali) si rivolge dunque direttamente alle dame presenti, destinatarie della festa, porgendo le scuse degli accademici in un salace monologo carico di doppi sensi e di un riposto allegorismo, che riecheggia la dedica decameroniana alle donne, e cioè la scelta di una cifra letteraria volgare e divulgativa, antilatina e anti-pedantesca:

Io vi veggio fin di qua, nobilissime donne, meravigliate di vedermivi così dinanzi in questo abito e, insieme, di questi apparecchio come se noi avessimo 
a farvi qualche comedia.Comedia non vi dovete pensare: ché, infin l'anno passato, voi poteste conoscere che l'Intronati avevano il capo ad altro che alle commedie; e poi vedeste, l'altro giorno, qual fusse intorno alle cose vostre l'animo loro e che non volevano più vostra pratica, né venirvi più dietro, come quelli che non gli piaceva più essere morsi, rimenati per bocca e tocchi fino al vivo da voi. E però abbruciarono, come voi vedeste, quelle cose che gli potevano far drizzare la fantasia e crescere l'appetito di voi e delle cose vostre. Ora vi voglio cacciar questa meraviglia dal capo. Questi Intronati, a dirvi ' 1 vero (e crediatemi, ch'io gli ho sentiti), si dolgono strettamente d'essere entrati in questo farnetico [...]. Quanto ha di bello il mondo, senza dubbio, è oggi in Siena; e quanto ha di bel Siena si truova al presente in questa sala. (Accademia degli Intronati, 2009, p. 34)

Il prestigio e l'eccellenza femminili resteranno una costante nel laboratorio drammaturgico intronatico, in cui si fissano i modelli vincenti del genere commedia volgare romanzesca e tragicomica, sia come cifra pedagogica e comunicativa (che presiede ad esempio ad un'intensa attività accademica di volgarizzamenti), sia come concreta pratica sociale e mondana, che vedrà molte dame della città partecipare attivamente alla vita del sodalizio anche come attrici, e persino, a metà ' 600 , fondare un'accademia sorella tutta al femminile, l'Accademia delle Assicurate, costola teatralmente molto attiva di quella intronatica.

In ambito cortigiano e accademico valgono dunque il prestigio e il patrocinio di un'élite femminile aristocratica, che pratica in proprio e contribuisce ad imporre la novità della rappresentazione drammatica, e che continuerà a coltivare uno sperimentalismo teatrale di avanguardia in regime protetto entro spazi privati, recitando, cantando, traducendo testi classici e scrivendone di moderni fino a '700 inoltrato. Questo tipo di teatro, dopo una trionfale fioritura cinquecentesca, sarà presto affiancato e sorpassato dal teatro commerciale dei professionisti e degli impresari, rivolto ad un vasto pubblico interclassista di spettatori paganti; ma, almeno in Italia, la pratica amatoriale performativa e musicale della veglia o della recita in villa o in salotto resisterà fino alle soglie dell'età moderna e poi si prolungherà nelle filodrammatiche amatoriali ottocentesche come spazio drammaturgico "di ricerca", l'unico in cui donne privilegiate per rango o cultura hanno qualche possibilità di misurarsi. 
Fra la fine del ' 500 e il ' 700 andare a teatro diventa una pratica diffusa e in crescita, che genera problemi di censura e di ordine pubblico, nuove regole di comportamento sociale e un enorme dibattito teorico intorno alla fascinazione del teatro, di solito cupamente anche se vanamente proibizionistico, dal fronte religioso (sia cattolico che protestante) come da quello politico. Nei corrales spagnoli, nei teatri elisabettiani e nelle sale francesi e italiane il problema sarà costantemente quello di separare i sessi e i diversi ceti sociali, per riparare le élites dalla promiscuità rissosa del parterre, in cui le poche donne si avventurano spesso in panni maschili oppure in maschera, e magari, se sono gentildonne, attraverso passaggi segreti, come quello che a Firenze collega Palazzo Vecchio al teatrino di Baldracca dove recitano i comici dell'arte.

Il travestitismo non è solo il topos per eccellenza della drammaturgia barocca, ma anche una diffusa pratica di sopravvivenza fisica, di autodifesa e di decoro sociale, costantemente quanto vanamente proibita dai vari legislatori. Nonostante tutto, però, il contagio del teatro è inarrestabile e penetra persino in convento, dove, grazie ai loro privilegi di sangue, sono le monache di buona famiglia che riescono spesso e volentieri a strappare margini di manovra e permessi di allestire spettacoli alle riluttanti autorità vescovili (una storia avvincente, più di costume che di spettacolo, di continue eccezioni alla regola). La massa del pubblico minaccioso e violento, appassionato e avido di emozioni, acquista intanto sempre più spazio e autorevolezza $\mathrm{e}$, molto lentamente, comincia anche a rincivilirsi. Da Lope in poi il suo gusto e il suo piacere diventano, nonostante tutto, un indicatore fondamentale, e inizia quel lento processo di transizione da un'estetica dell'oggetto a un'estetica del soggetto che trasforma l'esperienza teatrale in una questione di passione e di coinvolgimento emotivo sempre più individuale, riportando le donne al centro dell'attenzione, come bersaglio polemico ma anche, e soprattutto, come destinatarie ideali di un teatro sognato eccellente.

La rivoluzione emozionalistica dell'esperienza estetica teorizzata da Jean Baptiste Du Bos nel 1719 nelle sue Réflexions critiques sur la poésie et la peinture è accompagnata da un'aspra e affollata serie di recriminazioni contro la degenerazione degli spettatori contemporanei superficiali, edonisti e capricciosi, cioè, a parere di personaggi come Jac- 
ques Bénigne Bossuet o Carlo Gozzi (affini e consonanti pur a distanza di un secolo), sempre più femminilizzati. Quando in Francia, verso la metà del '700, si cercherà di portare un po' d'ordine nel caos dei teatri pubblici, cacciando i nobili dal palcoscenico e mettendo a sedere gli spettatori popolari del parterre per tenerli un po' più calmi, sono in molti ad opporsi a queste innovazioni (per esempio Diderot o Marmontel) proprio in base all'idea che sedute e comode ci stanno le donne (quelle aristocratiche delle balconate) e che il pubblico virile della sala ha il diritto/dovere di manifestare, stando in piedi, tutte le proprie reazioni di condivisione o di dissenso. A questo pubblico pigro e infantile e ai venali professionisti della scena si imputeranno topicamente le principali responsabilità del malcostume teatrale lamentato in coro da intellettuali settecenteschi anche di opposti schieramenti ideologici, mentre i grandi uomini di scena - da Metastasio a Riccoboni, da Marivaux a Goldo$\mathrm{ni}$ - si sforzeranno invece di interpretarne e assecondarne le istanze senza curarsi delle poetiche, e spesso guardando in particolare alle donne come sicure garanti del gusto che sta cambiando.

Le donne - in veste di personaggi dei nuovi drammi che liquidano l'immaginario cavalleresco, e di spettatrici competenti - balzano alla ribalta, diventano la nuova frontiera di ogni progressismo culturale e un campione fondamentale di riferimento estetico. Come al solito questo non presuppone affatto una loro equivalente emancipazione né un'autorevolezza reale: è soltanto una metafora culturale, che tuttavia innesca fatalmente anche una tendenziale trasformazione dei costumi. La querelle fra antichi e moderni è indissolubilmente connessa alla querelle des femmes: le relazioni familiari, l'amore, il matrimonio, le lacrime diventano materia di una drammaturgia nuova, che si svincola dalle gabbie del classicismo e abbandona le astruserie barocche proprio dichiarandosi ufficialmente indirizzata alle donne, meno colte ma più nä̈ves e ricettive dei maschi:

Le sensazioni devono essere più vivaci nelle donne di quello che non lo siano negli uomini; e ciò per la maggior delicatezza d'organizzazione; la immaginazione femminina e quella finissima grazia ch'elleno naturalmente hanno, sia raccontando, sia scrivendo, sia che vi dipingono gli oggetti al vivo, ne sono una prova bastante. Lo stile delle donne, per poco che sieno 
elleno dirozzate dalla educazione, in ogni lingua è composto di vezzi d'un tal genere che noi indarno cercheressimo di ritrovare. Da questo principio ne nasce che le passioni sieno anche più violente nelle donne, cosicché suprano le nostre, se non nella durata, almeno nella intensione. (Francioni, Romagnoli, 1993, p. 250)

Si tratta ancora di donne più immaginarie che reali, o meglio di donne reali sempre un po' ritoccate e corrette, fantasticate come lettrici voraci di romanzi (che in verità leggono anche gli uomini) con gli abbandoni e le estasi sospette raffigurate da tanti pittori rococò. Lettura e fornicazione sono scientificamente abbinate dalla medicina dell'epoca, e sui corpi femminili si consuma, come al solito, un'impropria e molto simbolica lotta fra opposti partiti per parlare d'altro (Serra, 2011, p. 66 e sgg). Non è particolarmente vero che il Settecento sia un secolo "femminista", come una serie di studi vanno dimostrando in questi ultimi anni; nella vita reale delle persone vince largamente «l'altro Settecento» di cui conosciamo le invincibili superstizioni e segregazioni (Guerci, 1987), tuttavia ci sono ora più donne in circolazione sulla scena pubblica e la loro visibilità è legata da vicino a questi due oggetti così in vista e così intrecciati fra loro: il romanzo e il dramma. Il loro inedito protagonismo come consumatrici ne fa ben presto anche delle produttrici: in molte cominciano timidamente a scrivere, a tradurre e a recensire. Nascono periodici dedicati a loro, come a Venezia il fortunato "La spettatrice" $\mathrm{o}$, più tardi a Milano "Il corriere delle dame" (1804); circolano le traduzioni di drammi francesi di Luisa Bergalli Gozzi, Maria Vittoria Ottoboni Serbelloni, Elisabetta Caminer; si intrecciano, in giro per l'Europa, importanti carteggi teatrali "al femminile", come quello fra madame Riccoboni e Garrick.

Carlo Gozzi esecra tutto questo: nelle Gare teatrali, una commedia allegorica scritta negli anni ' 50 in polemica col Teatro comico di Goldoni e ambientata in una immaginaria Ovadia, prende ferocemente di mira queste nuove esperte nei personaggi di due contesse stupide, vanesie e fanatiche - Favilla e Trombetta - che patrocinano opposte claques teatrali (i chiaristi e i goldonisti); liquida in modo sprezzante la giovane Elisabetta Caminer, che gli fa concorrenza traducendo drammi francesi, come «ragazza macchinetta» manovrata da altri (maschi 
naturalmente), e fiuta da questi segnalil'avvento di una prossima apocalisse sociale:

Le femmine sbucarono tosto da' loro alberghi, sfrenate come le antiche baccanti, e gridando libertà, imbrogliarono tutte le strade, scordarono figli, figlie, servi, lavori ed economia, e colla testa fumante, unicamente occupata nelle mode, nelle emulatrici frivole invenzioni, nel profondere per l'appariscenza, ne' spassi, ne' giuochi, negli amori, nel civettare, abbandonate a' loro capricci, fomentati da' lor consiglieri filosofi. I mariti non ebbero più coraggio d'opporsi alla desolazione del loro onore, delle loro sostanze, delle loro famiglie, del mal esempio alla figliolanza, per timore d'esser macchiati dal vocabolo "pregiudizio". (Gozzi, 2006, I, p. 369)

Nei fatti andare a teatro - a Venezia lo si fa in maschera il che moltiplica i rischi - è una sfida di libertà che può avere il sapore di una trasgressione da reprimere. Nella sua Riforma all'insegna della moralità delle scene, Goldoni si proclama autore di commedie a cui i padri di famiglia possono condurre senza problemi mogli e figlie:

Per nostra consolazione, non solo è sbandito qualunque reo costume nelle persone, ma ogni scandolo dalla scena. Più non si sentono parole oscene, equivoci sporchi, dialoghi disonesti. Più non si vedono lazzi pericolosi, gesti scorretti, scene lubriche, di mal esempio. Vi possono andar le fanciulle, senza timor d'apprendere cose immodeste o maliziose. (Goldoni, 1943, p. 1093)

Ma all'interno dei suoi componimenti - peraltro non sempre così morigerati come egli dichiara - il diritto femminile al teatro è spesso ambiguamente censurato perché presuppone sfumature di irrequietezza e di ribellione: ci sono le spensierate Morbinose che vanno a divertirsi in libertà, lasciando i mariti a casa nella licenza del carnevale, o la spregiudicata donna Lucrezia delle Donne gelose, una vedova che vive di espedienti, di spettacoli e di ridotti, pur restando abilmente entro i confini della rispettabilità; ci sono le smaniose mogli dei Rusteghi, che rimpiangono la privazione degli spettacoli, con la sola eccezione (forse soltanto carnevalesca) di siora Felice, che trascina spavaldamente all'opera marito e cavalier servente, e nella Casa nova c'è la giovane e frivola Cecilia, che si vanta di avere un palco privato, e di andarci quando 
vuole e con chi vuole, ma che ha torto, e dovrà cambiare condotta su tutti i piani.

Goldoni è un emancipazionista molto cauto, spesso crudele con le sue eroine, che, risolto l'intreccio, torneranno ai destini piuttosto malinconici che i suoi ambigui lieti fine lasciano ampiamente intravedere. Come tutti mette alla berlina le femmes savantes sulle scene in nome di un ordine familiare e sociale ovviamente patriarcale, e tuttavia è attratto in società dalle nobildonne colte, e cerca sempre di sfruttarne il patrocinio teatrale, a Venezia, come a Roma, a Milano come a Parigi (Pieri, 2016). Costoro non sono né lavandaie né mercantesse, per il momento, ma appunto signore aristocratiche, che incarnano, sul piano mondano e sociale, un'avanguardia di gusto naturalmente solidale con la sua battaglia di autore, e che gli offrono un appoggio prezioso per riscattarsi dall'ossequio sornione o burlesco dovuto alle temibili tifoserie teatrali, e l'opportunità di un'interlocuzione alla pari. Il dato emerge con chiarezza (seppure in forme cifrate e dissimulate) se ci si addentra nel labirinto dei paratesti critici e delle sue oculate strategie editoriali nei confronti di queste signore, libere di disporre di sé, che leggono, scrivono, frequentano il teatro, e anche lo praticano in prima persona in salotto, in villa, o in accademia nei circoli arcadici emiliani e lombardi; un'enclave privilegiata di intoccabili (la cui vita privata è spesso irregolare e talvolta persino scandalosa) a cui Goldoni si rivolge soprattutto in alcuni momenti-chiave del suo lavoro, quando si tratta di valorizzare adeguatamente dei passaggi cruciali (La donna di garbo, Il teatro comico), fronteggiare delle crisi (La vedova spiritosa), affrontare svolte impreviste o rilanci insidiosi (trilogia persiana, Statira).

Esse hanno margini di manovra proibiti a Rosaura o a Bettina, e anche a siora Felice o a Giacinta: il loro sesso è una referenza specifica e un valore aggiunto, a cui il drammaturgo, così cauto in scena, si rivolge nei libri con cordiale franchezza. Lo scambio avviene ufficialmente in pagina, nei componimenti encomiastici, nelle dedicatorie, nelle lettere, che proseguono dialoghi intrecciati nelle conversazioni private in villa e in accademia, e nella pratica condivisa di uno sperimentalismo teatrale fatto di traduzioni e di recite amatoriali di villeggiatura, di collegio e di convento. Tutti motivi ampiamente esplorati dalla critica, 
che confermano il ritratto storicamente ovvio di un Goldoni paternalista con le popolane, severo con le borghesi, più che indulgente con le nobili e fedifrago con le attrici.

La storia continua nelle sale teatrali ottocentesche, sempre più interclassiste, dove una borghesia, ormai culturalmente emancipata e rincivilita, austera e moralistica, si accredita come anima autentica del nuovo pubblico italiano, portandosi appresso mogli e figlie, castigate e sottomesse ma pur sempre seducenti e pericolose:

È atto inurbano il fissare con soverchia intensione le signore che trovansi ne' palchetti, nelle logge o nelle file della platea, obbligandole spesso così a rivolgere altrove la testa, per conservare le apparenze almeno della tanto pregiata modestia, e togliendo loro il godimento di parte dello spettacolo, o il piacere di conversare con persone vicine e di propria confidenza. (Savonarola, 2012, p. 34)

Offende pure l'immaginazione del sesso forte il vedere in teatro qualche signora scoperta più che nol comportino il buon costume e la decenza. Una vezzosa sposina pavoneggiavasi in un palchetto delle sue morbide spalle, che scoperte fino disotto dell'ascella, appagando lautamente lo sguardo de' circostanti, toglievano alla loro immaginazione il diletto proveniente dal desiderio. Non mi accadde mai di sentire altrettanto contro qualsiasi donnicciuola da trivio. (Ibidem, p. 61)

In platea e nei palchetti, metonimia della nazione futura, contano, almeno in teoria, i singoli spettatori, selezionati dall' egualitarismo romantico della sensibilità e del cuore, ma le differenze di status sono in tutta evidenza. Andare a teatro, per uomini e donne, è ormai un aspetto fondamentale della socialità, onnipresente infatti nei romanzi ottocenteschi da Stendhal a Balzac, e da Tolstoj a Verga; la platea - descritta da Carlo Dossi come un panopticon da cui si può osservare l'intera società come «merce umana» - è la vetrina ufficiale, ma il foyer, in cui ci si mescola fumando e conversando, è uno spazio nevralgico: a fine '800 all'Opéra Garnier di Parigi è diventato infatti molto più ampio della platea. Le donne sono, al solito, al centro dell'attenzione come corpi da guardare. Ma qui ci dobbiamo arrestare. 


\section{BIBLIOGRAFIA}

Accademia degli Intronati (2009). Gl'Ingannati, a cura di M. Pieri. Corazzano: Titivillus.

Cavallo, G., \& Chartier, R. (Eds.). (1998). Storia della lettura. Bari: Laterza. Coppo, A.M. (1968). Spettacoli alla corte di Ercole I. In Contributi dell'Istituto di Filologia Moderna. Serie Storia del teatro (vol. 1, pp. 30-59). Milano: Pubblicazione dell'Università Cattolica del Sacro Cuore.

Fabiano, A. (2019). Haine du théâtre, haine des femmes : fascination et censure dans la réflexion théorique et dans la pratique théâtrale italiennes au XVIIe siècle. Littératures Classiques, 99, 79-91.

Francioni, G., Romagnoli, S. (Eds.). (1993). «Il Caffè» (1764-1766). Torino: Bollati Boringhieri.

Goldoni, C. (1943). Il teatro comico. In Idem, Tutte le opere, a cura di G. Ortolani (vol. 1, pp. 1039-1105). Milano: Mondadori.

Gozzi, C. (2006). Memorie inutili, a cura di P. Bosisio, V. Garavaglia, voll. 2. Milano: Led.

Guerci, L. (1987). La discussione sulla donna nell 'Italia del Settecento. Aspetti e problemi. Torino: Tirrenia.

Manguel, A. (2009). Una storia della lettura. Milano: Feltrinelli.

Pieri, M. (2016). «Pour plaire au public, il faut commencer par flatter les dames» (Mémoires, II, XL). Goldoni e le nuove frontiere del pubblico. In C. Berger \& F. Coletti (Eds.). Les figures du féminin en rupture» à Venise: courtisanes, actrices, épouses, servantes et putte du XVIe au XVIIIe siècle (pp. 229-259). Toulouse: Université Toulouse Jean Jaurès. Sanuto, M. (1879-1902). I diarii (MCCCCXCVI-MDXXXIII) dall'autografo Marciano Ital. Cl. VII Codd. CDXIX-CDLXXVII. Venezia: Visentini.

Savonarola, G. (2012). Galateo dei teatri, a cura di M. Sirtori. Milano: Lubrina. Serra, F. (2011). Le brave ragazze non leggono romanzi. Torino: Bollati Boringhieri.

Riassunto: La spettatrice aristocratica di corte o di accademia, agli inizi dell'età moderna, nobilita con la sua presenza il nuovo teatro classicistico, ed è spesso destinataria privilegiata di omaggi drammaturgici e di saggi di poetica; essa è tuttavia molto diversa dalle spettatrici reali che cominciano a frequentare i teatri pubblici a pagamento nell'Europa del XVI e XVII secolo. Le donne a teatro, sulle scene e in platea, rappresentano per le autorità religiose cattoliche e protestanti un problema e uno scandalo da reprimere e censurare; la loro presenza tuttavia cresce e si afferma nel ' 700 , quando cominciano a diventare le depositarie principali del buon gusto e del sentimento. Il saggio analizza le due facce di questa storia.

Parole chiave: spettatrice, censura, patrocinio, commedia, sensibilità 